\title{
QUELQUES DONNEES HISTORIQUES CONCERNANT LA SEXOLOGIE
}

\author{
Robert Gellman* et Claire Gellman-Baroux**
}

\author{
* Psychiatre des Hôpitaux de Paris, \\ ** Psychologue secrétaire du cours de sexologie de l'hôpital Necker de Paris, 3, rue Copernic 75116 Paris.
}

\section{SOME HISTORICAL FACTS ON} SEXOLOGY. With the exception of forensic medicine and psychiatry, medicine has not historically been substantially influenced by sexology. After Freud and psychoanalysis grappled with sexual facts, a sociological phase followed. However, it was not until Masters and Johnson that sexology was established as an independent science. Now; thanks to the East Coast of the United States, sexological treatments are numerous, including the corporeal approach and the use of audiovisual aids. The problem now is to establish therapeutic strategies. Key words : Psychoanalysis, sociology, Masters and Johnson, Therapeutic strategies. Andrologie, 1992, 2:32-34.

Les réticences de la Médecine face à l'étude des problèmes sexuels sont bien résumés par l'un des plus anciens sexologues français : Grimaud de Caux (6). Dès 1837, cet auteur exprimait les difficultés qu'il avait rencontrées : "Quand nous avons manifesté, pour la première fois, le projet de publier une Histoire de la génération de l'homme, nos amis nous ont représenté la difficulté particulière de cette entreprise, qui consistait en effet à disserter à haute voix, avec convenance et utilité, sur un sujet qu'on ose seulement nommer entre les dents, comme dit Montaigne. Quelques-uns même, pour nous détourner, allèrent jusqu'à prétendre que son exécution était dans le cas de compromettre le peu de valeur scientifique ou littéraire que nous avions péniblement acquise. Nous avions beau répondre que les personnes étaient fort rares qui, à vingt ans, ignoraient innocemment quelquesunes de ces choses desquelles il est reconnu et admis qu'il ne faut parler qu'à voix basse ; on convenait de la vérité du fait, et l'on persistait néanmoins à blâmer notre dessein".

\section{SEXOLOGIE ET MEDECINE}

Leurs rapports ont donc toujours été empreints de beaucoup de réserve. Classiquement, le rôle du médecin étant uniquement de traiter la douleur et les affections organiques caractérisées, la pathologie fonctionnelle et tout ce qui touche la sexualité était rejeté de son champ. Seule la médecine légale s'intéressait à la description des comportements sexuels pathologiques.

G. Abraham écrivait en 1974 (1) : "Si une sexologie médicale commence à exister depuis le siècle dernier, il s'agit d'une sexologie essentiellement descriptive, une description des phénomènes sexuels groupés avec des critères plus ou moins nosographiques".

Parmi les précurseurs de la sexologie, il faut citer Von Krafft-Ebbing, Havelock Ellis, Magnus Hirschfeld, Auguste Forel. "La thérapie envisagée ne pouvait être que symptomatique... la sexologie restait toujours un fait assez marginal de la médecine" (1).

\section{SEXOLOGIE ET PSYCHIATRIE}

Leurs relations sont historiquement très anciennes et très nombreuses. C'est ainsi que le traité de Pathologie Mentale de Gilbert Ballet (2), publié en 1903, aborde dans différents chapitres: - du sexe et des fonctions sexuelles dans les causes déterminantes des affections mentales (P 29).

- des affections des organes génitaux génératrices d'affections mentales (P 51).

- des fonctions génésiques (P 116).

- des idées érotiques dans la description séméiologique des diverses idées délirantes (P 266).

- des délires systématisés érotiques, en particulier l'Erotomanie d'Esquirol (P 573).

- des perversions génitales (P 1521) et de la folie puerpérale (P 1541).

\section{SEXOLOGIE ET PSYCHANALYSE}

Leurs rapports sont très complexes. Sigmund Freud représente une seconde étape dans l'histoire de la sexologie. Il s'attaque à la dynamique des faits sexuels. Il crée une sexologie psychiatrique : Selon Abraham (1, p 22) "C'est une compréhension qui, tout en essayant de dresser des théories importantes dans le domaine de la sexualité, limite la compréhension sexologique fondamentale à ses liens avec les manifestations névrotiques". Ce même auteur résume ainsi les points fondamentaux de la démarche freudienne :

1 - L'impulsion sexuelle a un rôle déterminant dans le développement psycho-affectif de chaque individu.
2 - L'impulsion sexuelle est active dès l'enfance. Freud attache, comme nous le savons, une importance fondamentale à la sexualité infantile. C'est ainsi qu'il écrit dans ses "trois essais sur la théorie de la sexualité" parus en 1905 et remaniés aux cours des diverses éditions successives jusqu'en 1924. "Si nous approfondissions les manifestations sexuelles de l'enfant, nous découvririons les traits essentiels de la pulsion sexuelle, nous comprendrions l'évolution de cette pulsion, et nous verrions comment elle puise à des sources diverses" $(4, \mathrm{p} 25)$.

Il s'attache particulièrement à l'étude de "l'amnésie infantile" dénommant ainsi "le voile épais" qui couvre les six ou huit premières années de la vie qui crée "pour chacun de nous dans l'enfance une sorte de préhistoire en nous cachant les débuts de la vie sexuelle" (4). "Ne serait-on pas conduit à penser que l'amnésie infantile elle-même n'est pas sans rapport avec la sexualité de l'enfant ?" se demande-t-il (4).

3 - "La sexualité intègre progressivement des éléments partiels dont l'harmonie permettra l'expression d'un comportement sexuel mûr et satisfaisant" (1).

4 - "La sexualité détermine des conflits inhérents au développement lui-même et y participe. De cela il résulte une potentialité conflictuelle permanente rattachée à l'impulsion sexuelle et l'importance étiologique prépondérante que la sexualité acquiert dans l'éclosion et le maintien des névroses et d'autres atteintes psychopathologiques" (1).

De tous ces points, il résulte que la sexologie ayant ses racines dans le développement psychosexuel de l'enfant, pour le psychanalyste le traitement ne pourra être qu'une thérapie de l'individu.

De nombreux psychanalystes se sont attachés à la suite de Freud à déterminer les aspects psychodynamiques de la sexualité. On peut citer parmi les plus importants : Karl Abraham, Férenczi, Jones, Hélène Deutsch, Marie Bonaparte, Mélanie Klein...

\section{SEXOLOGIE ET SOCIOLOGIE L'étape Kinsey}

Alfred Kinsey et son équipe $(7,8)$ ont introduit en sexologie l'outil statistique. Le premier volume de leur enquête est paru en 1948. Son 
grand mérite a été de permettre une évaluation plus précise du comportement sexuel supposé normal, et d'éliminer un certain nombre de préjugés, d'où une perspective thérapeutique de type social.

Un certain nombre de faits statistiques ont bouleversé les connaissances antérieurement admises. Les données les plus controversées ont concerné :

1 - la sexualité infantile. D'après une enquête portant sur 317 cas :

- un tiers des enfants mâles découvriraient l'orgasme avant l'âge de 12 mois et la moitié avant 7 ans. En dehors de l'éjaculation qui fait défaut, cet orgasme correspond à celui de l'adulte.

- $39 \%$ des garçons de 12 ans reconnaissent avoir pratiqué une quelconque activité sexuelle sur autrui contre $10 \%$ des filles du même âge.

- avant 15 ans, $82 \%$ des garçons se sont masturbés contre $20 \%$ de filles seulement. A 20 ans, les chiffres respectifs sont $92 \%$ et $82 \%$.

- Kinsey rapporte que $80 \%$ des femmes se souvenaient d'avoir été caressées au moins une fois par un homme entre 5 et 10 ans.

2 - l'homosexualité. Dans les statistiques de Kinsey, $33 \%$ des hommes contre $13 \%$ des femmes auraient connu l'orgasme au cours d'une expérience homosexuelle. $50 \%$ des individus hommes et femmes ont eu des rapports homosexuels : seulement $8 \%$ d'entre eux s'y livrent exclusivement pendant plus de 3 ans.

3 - Les conduites hétérosexuelles de ladolescent:

- $90 \%$ des femmes s'étaient livrées à des caresses érotiques pré-conjugales. Parmi elles, $30 \%$ avaient éprouvé l'orgasme.

- $87 \%$ des femmes qui ne se sont jamais masturbées n'accèdent pas à l'orgasme au cours de la première année de mariage contre $13 \%$ seulement des femmes habituées à se masturber.

\section{4 - La sexualité de l'adulte :}

- La femme a deux fois moins de fantasmes érotiques que l'homme. $69 \%$ des hommes contre $31 \%$ des femmes.

- $95 \%$ des hommes reconnaissent pratiquer des stimulations tactiles des organes génitaux de leur partenaire contre $91 \%$ des femmes.

- $60 \%$ des hommes de formation universitaire s'adonnent aux caresses bucco-génitales.

- La fréquence moyenne des rapports passe de 3,21 par semaine dans le groupe des 16 à 20 ans à 1,80 dans le groupe des 50 ans et 0,9 dans celui des 70 ans. Les données de Kinsey sont déjà relativement anciennes.
Données sociologiques plus récentes. L'étude de la sexualité en 1991 permet un certain nombre de constatations.

Il y a, tout d'abord, un contraste entre la libération sexuelle dont chacun peut bénéficier, et la permanence des difficultés sexuelles que présentent nos patients, même les plus jeunes. En effet, les problèmes au niveau des interdits infantiles et odipiens restent identiques.

L'action des mass media et de l'éducation sexuelle a des limites.

Les sujets qui en bénéficient vraiment sont surtout ceux qui n'auraient, de toutes façons, pas présenté de difficulté importante.

En règle générale, l'information sexuelle n'a pas été apportée aux sujets par leurs parents, ce qui rend très difficile tout dialogue sur ce thème entre les deux générations. Des cours d'information sexuelle sont actuellement organisés, mais ils sont le plus souvent très mal intégrés.

"Des éléments divers provenant de lectures, de bribes de conversations d'adultes, de séquences cinéma-tographiques, d'entretiens avec des camarades mieux informés, participent à la compréhension globale de la sexualité"... "C'est en fait une prise de conscience progressive, en relation avec l'évolution maturative de l'enfant" (5).

De nouveaux problèmes surgissent, en relation avec la généralisation de la contraception.

La liberté sexuelle de la femme provoque chez l'homme une nouvelle angoisse : celle de voir sa compagne effectuer d'autres expériences sexuelles sans être retenue par le risque d'une grossesse. On assiste ainsi à l'apparition d'une nouvelle source d'angoisse, celle de ne pas être satisfaisant, qui favorise tous les troubles sexuels.

"La libération sexuelle de la femme, et sa revendication du droit au plaisir, sont parfois vécues par son partenaire comme une menace d'infidélité et une exigence de performance. Il existe à cela une contrepartie : le rôle actif dans la relation sexuelle n'est plus l'apanage de l'homme, qui regagne ainsi en jouissance ce qu'il perd en sécurité" (5).

\section{Sexualité des jeunes.}

D'une enquête sociologique exhaustive de Jean Duvignaud (3) ressortent les faits suivants : - l'investissement de la libido dans une idéologie politique ou syndicale semble jouer un rôle de substitut, et entraine une diminution de la sexualité individuelle. "Au moment où la liberté réelle et pratique est plus grande, la revendication abstraite pour la liberté de l'humanité remplace les contraintes traditionnelles, et agit comme une véritable répression".
- Les expériences de sexualité de groupe, lorsqu'elles sont réalisées, sont souvent mal acceptées, ef entraînent de ce fait une brisure au sein des couples qui avaient une existence antérieure.

- L'importance des essais prénuptiaux est nettement soulignée.

- Le mariage n'est guère contesté par les jeunes, surtout par les femmes. Mais il est reporté à un moment où les sujets désirent la stabilité dans les relations sexuelles avec un seul partenaire. A une époque où les rites initiatiques ont presque disparu, le mariage et la naissance des enfants apparaissent comme un substitut d'expérience intiatique marquant le passage de l'adolescence à l'âge adulte. Duvignaud (3) écrit ainsi :

"La plus grande part de nos réponses renvoie à une coutume sexuelle sentimentale qui culmine sur l'existence du couple".

- Le sentiment amoureux parait devoir compléter de façon essentielle la sexualité, pour les partisans d'une vie de couple.

\section{L'APPORT DE MASTERS ET JOHNSON}

C'est grâce à leur contribution que la sexologie s'est constituée comme une science autonome. Masters et Johnson ont entrepris leur recherche sur la sexualité humaine en 1954. Dans un premier temps, ils se sont consacrés à l'étude des réactions sexuelles (9). Ils ont étudié, à la Fondation de Recherche sur la Biologie de la Reproduction de Saint-Louis, les réactions de l'être humain lors de l'acte sexuel. Ils ont pu ainsi mesurer celles de 694 hommes et femmes choisis dans des milieux et des âges différents. Ils ont mis en évidence le cycle des réponses sexuelles chez la femme et chez l'homme jeunes puis âgés.

A partir de ces données, ils se sont ensuite intéressés aux troubles sexuels et ont mis au point leurs traitements de couples (10). En effet, leur patient est le couple lui-même, car Masters et Johnson pensent que c'est la relation entre les conjoints qu'il faut traiter, et non les partenaires pris isolément.

Ce couple est pris en charge par un couple de thérapeutes qui agit comme un catalyseur de la communication. Des exercices progressifs de sensibilisation sont prescrits aux couples qui doivent les pratiquer en privé.

Ainsi, ils ne permettent pas à l'individu souffrant de troubles sexuels de rester seul avec ses fantasmes et ses idées préconçues sur son partenaire. Ils exigent que celui-ci soit présent dans la plénitude de sa réalité (1).

Dans la méthode de Masters et Jonhson, les phénomènes transférentiels existant entre les patients et les thérapeutes sont réduits au 
minimum et négligés. Leur méthode entre cependant dans le cadre des psychothérapies : l'échange thérapeute-patient y est purement verbal ; la revalorisation sensorielle n'intervient qu'au niveau du couple.

\section{LES TECHNIQUES CALIFORNIENNES}

Sur la côte californienne, en particulier à l'Université de San Francisco, d'autres expériences thérapeutiques se sont développées. Dans les techniques californiennes, le contact corporel s'établit entre thérapeutes et patients et sinsère dans une vaste gamme de traitements. En ce qui concerne l'abord corporel, différentes techniques de massage sont utilisées, ainsi que des traitements par la nudité et le toucher. Sont encore employées les diverses méthodes de relaxation, le yoga, la bio-énergie issue des travaux de Wilhelm Reich, etc...

D'une façon générale, les thérapeutes s'impliquent dans les traitements; ils n'hésitent pas par exemple à parler de leur propre sexualité, afin de diminuer l'inhibition de leurs patients. En plus de l'abord corporel, les méthodes audiovisuelles sont largement utilisées, avec une projection intensive de films à contenu sexuel. Tous les comportements sexuels (hétérosexualité, homosexualité, masturbation, etc...) y sont représentés. Ces films ont un intérêt particulier pour le traitement des sujets psychosomatiques, dont l'activité fantasmatique est généralement pauvre. Le psychodrame, les jeux de rôles, les thérapies de groupes (en particulier groupes de femmes anorgasmiques) sont également pratiqués.

\section{CONCLUSIONS}

G. Abraham s'interroge sur le devenir de la sexologie (1). Elle s'orientera peut-être dans l'avenir "en deux branches complémentaires, l'une d'obédience scientifique et médicale, concentrée sur les faits, le besoin indestructible de guérir, et l'autre plus libre, plus irrationnelle, plus hardie, qui continuera à se demander le pourquoi de l'existence du sexe, et sa vraie signification".

Dans un domaine aussi complexe que celui de la sexualité humaine, on ne peut négliger ni les facteurs organiques, ni les causes psychologiques, non plus que les facteurs relationnels ou socio-culturels. Nous n'avons pas à imposer à nos patients liidée préconçue d'une sexualité normalisée, mais en les aidant à prendre conscience et à accepter leurs fantasmes, en les aidant à faciliter la communication dans leur couple, en les revalorisant dans leurs corps, en rétablissant un fonctionnement plus harmonieux au point de vue organique, nous leur permettons de découvrir et d'assumer "leur" sexualité.

\section{REFERENCES}

1 - Abraham G., Pasini W. : Introduction à la Sexologie Médicale Payot 1974 p. 22 - 25 - 27.

2- Ballet G.: Traité de la Pathologie Mentale. Paris, Doin, Edition 1903

3- Duvignaud J. : La planète des jeunes. Edition Stock 1975

4 - Freud S. : Trois essais sur la théorie de la Sexualité. Edition N.R.F. 1962.

5- Gellman R. : La Sexualité des Adolescents. Cahier de Sexologie Clinique. Vol. 1. no 4 p. 403
6- Grimaux de Caux G.: $\quad$ Physiologie de l'espèce. Histoire de la génération de l'homme. Librairie Encyclographique 1837 p. VII

7 - Kinsey A., Martin N., Pomeroy N., Wandell D. : Sexual behaviaour in the human male. W. $3 \&$ Sanders Company 1948. Traduction Française aux Editions du Pavois 1948.

8 - Kinsey A., Abhard B., Llydée A., Pomeroy N., Wandell D. : Sexual behaviour in the human female W. \& Sanders Company 1953.

9 - Masters W. H., Johnson V. E. : Les réactions Sexuelle. Robert Laffont 1968.

10 - Masters W. H., Johnson V.E. : Les mésententes sexuelles et leur traitement. Robert Laffont, 1971.

RESUME : En dehors de la médecine légale et de la psychiatrie, la médecine a été peu concernée sur le plan historique par la sexologie. Freud et la psychanalyse se sont attaqués à la dynamique des faits sexuels. L'étape suivante a été sociologique, mais il a fallu attendre Masters et Johnson pour que la sexologie se constitue comme science autonome. Actuellement, grâce à l'apport de la côte Est des Etats-Unis, les modes d'abord sont multiples, incluant l'approche corporelle et l'utilisation de l'audio-visuel. Le problème présent est celui de la mise en place de stratégies thérapeutiques. Mots clés : Psychanalyse - Sociologie - Masters et Johnson - Stratégie thérapeutique. Andrologie, 1992, 2 : 32-34. 